\title{
The central star of the planetary nebula N66 in the Large Magellanic Cloud: A detailed analysis of its dramatic evolution $1983-2000^{\star, \star \star}$
}

\author{
W.-R. Hamann ${ }^{1}$, M. Peña ${ }^{2}$, G. Gräfener ${ }^{1}$, and M. T. Ruiz ${ }^{3, \star \star \star ~}$ \\ 1 Professur Astrophysik, Universität Potsdam, Am Neuen Palais 10, 14469 Potsdam, Germany \\ 2 Instituto de Astronomía, Universidad Nacional Autónoma de México, Apdo. Postal 70-264, 04510 México, DF, México \\ 3 Depto. de Astronomía, Universidad de Chile, Casilla 36-D, Santiago, Chile
}

Received 31 March 2003 / Accepted 11 July 2003

\begin{abstract}
The central star of the planetary nebula N 66 (alias WS 35, SMP 83 and HV 5967) in the Large Magellanic Cloud enhanced its brightness dramatically in 1993 and 1994. Within the subsequent four years it returned to the previous level. Its spectrum resembles that of a Wolf-Rayet star of the nitrogen sequence (WN4.5). We monitored the object intensively from ground and with the Hubble Space Telescope. Now we present the complete set of spectroscopic observations from the different epochs before, during and after the brightness outburst of $\mathrm{N} 66$. The stellar spectra from the different epochs are analyzed in detail by means of most advanced non-LTE models for expanding stellar atmospheres. The main results are: the luminosity, $\log L / L_{\odot}=4$.6, before and after the outburst is exceptionally high for a central star of a planetary nebula. During the outburst in 1994, it even climbed up to $\log L / L_{\odot}=5.4$ for about one year. The effective temperature of about $112 \mathrm{kK}$ remained roughly constant, i.e. the luminosity mainly increased because of a larger effective stellar radius. The mass loss rate increased from $10^{-5.7} M_{\odot} \mathrm{yr}^{-1}$ in the quiet state to $10^{-5.0} M_{\odot} \mathrm{yr}^{-1}$ during the outburst. The chemical composition of the stellar atmosphere is that of incompletely $\mathrm{CNO}$-processed matter: it is dominated by helium with a rest of hydrogen, nitrogen being slightly enhanced and carbon strongly depleted. We extensively discuss possible scenarios for the nature and evolutionary origin of $\mathrm{N} 66$, which should explain the exceptional stellar parameters, the atmospheric composition, the outburst mechanism, and the existence of the bipolar nebula which was ejected only a few thousand years ago and contains about 0.6 solar masses of hydrogen-rich matter. If being a single star, N 66 might be (i) a low-mass star after the Asymptotic Giant Branch, as usually adopted for central stars of planetary nebulae, (ii) a massive, i.e. non-degenerate star, or (iii) a merger produced from two white dwarfs. Although there are no direct indications for binarity, we alternatively discuss whether N66 might be (iv) a massive star which lost its hydrogen envelope in a recent common-envelope phase with a less massive companion, or (v) a white dwarf accreting mass from a companion with a high rate. None of the scenarios is free of any contradiction to at least one of the observational facts. However, the binary scenarios pose less severe problems. If N 66 is a white dwarf accreting matter in a close-binary system, its present accretion rate would bring it to the Chandrasekhar limit within a few hundred thousand years. Thus N 66 might be a candidate for a future type Ia supernova explosion in our cosmic neighborhood.
\end{abstract}

Key words. stars: winds, outflows - stars: mass-loss - stars: Wolf-Rayet - stars: evolution planetary nebulae: individual: LMC-N66

Send offprint requests to: W.-R. Hamann,

e-mail: wrh@astro.physik. uni-potsdam.de

* Partly based on observations with the NASA/ESA Hubble Space Telescope performed at the STScI which is operated by the Association of Universities for Research in Astronomy, Inc., under NASA contract NAS 5-26555.

$\star \star$ Partly based on observations with the International Ultraviolet Explorer (IUE).

$\star \star \star$ Visiting Astronomer at Cerro Tololo Inter-American Observatory, which is operated by the Association of Universities for Research in Astronomy, Inc. under cooperative agreement with the NSF.

\section{Introduction}

The planetary nebula N 66 (alias WS 35, SMP 83 and HV 5967) in the Large Magellanic Cloud (LMC) is identified first in a survey by Henize (1956). Optical spectroscopy (Monk et al. 1988; Peña \& Ruiz 1988) revealed its high excitation type. Dopita et al. (1993), in revision of earlier nebular analyses, estimated the effective temperature of the central star to $170 \mathrm{kK}$, and its bolometric luminosity to as much as $\log L / L_{\odot}=4.4$ which is exceptionally high for a central star of a planetary nebula.

Even more attention was attracted by N 66 when TorresPeimbert et al. (1993) reported changes in the optical spectrum of the central star, which clearly developed Wolf-Rayet type 


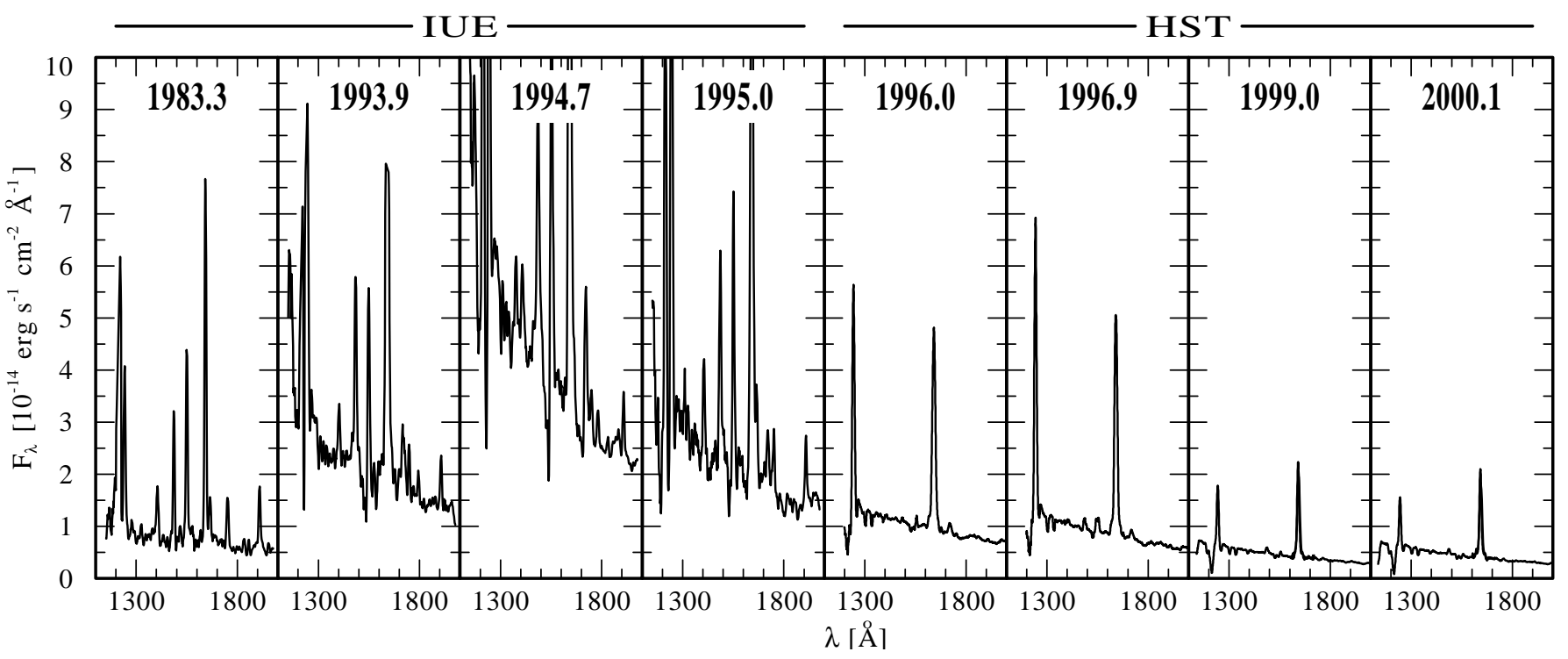

Fig. 1. The UV spectrum of N66 at different epochs, showing the dramatic rise and fall of the stellar flux. Note that nebular emission strongly contaminates the IUE observations, but is effectively suppressed by the small apertures of the HST spectrographs.

emission lines. Applying the classification scheme for massive Wolf-Rayet stars of the nitrogen sequence, Peña et al. (1994, 1995) assigned the spectral type WN 4.5 to the central star of N 66 - another exceptional property of that object since central stars with Wolf-Rayet spectra use to display carbon-rich (WC-type) composition.

In 1993 and 1994 the brightness of the N 66 central star rose dramatically, and then dropped again to its previous level within a few years. During this whole event we monitored the object intensively from ground and space, aiming at spectroscopic data with broad wavelength coverage. Between December 1995 and February 2000 the Hubble Space Telescope (HST) was employed four times for spectroscopic observations of N 66, exploiting HST's unique spatial resolution for isolating the stellar spectrum from the bright nebular emission.

A preliminary analysis of the outburst was presented by Peña et al. (1997, hereafter Paper I). At that time, we restricted ourselves to model only the change of continuum brightness, not yet investigating the time-dependent line spectra. The brightness change could be reproduced by assuming a mass-loss outburst, while all other parameters including the luminosity are kept constant. As we will see below, we cannot hold up this interpretation after a thorough analysis of all existing data, especially considering the detailed spectra from the different epochs.

The outburst of N 66 observed in 1994 might not have been the first one. Nail \& Shapley (1955) reported irregular variability by 0.9 mag in photographic brightness of the object (i.e., star plus nebula), while in the subsequent decades no variations could be detected.

Not only the central star, but also the nebula of N66 has been studied with the help of HST observations. Narrowband images retrieved from the HST archive were presented by Vassiliadis (1996). Especially one exposure, obtained after HST's spherical aberration was corrected with COSTAR, nicely displays the complex morphology of the nebula. Previous, ground-based spectroscopic studies of the nebular kinematic were refined recently by Peña et al. (2003) on the basis of high-resolution long-slit spectra obtained with the STIS spectrograph on board the HST.

The evolutionary status of N 66 is debated since the brightness outburst has been detected. In the beginning it was speculated (Peña et al. 1994) that N 66 might have been caught during a "very late thermal pulse", similar to a few other stars (like Sakurai's object, e.g. Asplund et al. 1999) which show a rapid development. Alternatively, we proposed in Paper I that a violent atmospheric instability might have caused a dramatic redistribution of the spectral energy distribution.

In the following section we present the complete set of spectroscopic observations available to us from the different epochs before, during and after the brightness outburst of N 66 . Our models, being of the most advanced state of the art for expanding non-LTE atmospheres, are described in Sect. 3, and applied for a detailed analysis of the whole spectroscopic data in Sect. 4. A comprehensive discussion (Sect. 5) is mainly devoted to the different scenarios which might explain the nature and evolutionary origin of this unique object.

\section{The observations}

Spectra of N 66 are available to us since 1983, when this object has been observed for the first time with the International Ultraviolet Explorer (IUE) satellite. Ground based observations, obtained with the 4-m telescope at the Cerro Tololo InterAmerican Observatory (CTIO) or the 3.6-m telescope at the European Southern Observatory (ESO) were taken since 1990, shortly before the central star started to increase its brightness. Since the end of 1995, the development of N 66 was monitored by us with the HST and ground-based telescopes.

The whole set of observations employed for the present study is compiled in Table 1. Spectra taken within a couple of 
Table 1. Log of observations.

\begin{tabular}{|c|c|c|c|c|c|c|}
\hline Epoch & Instrument & Image ID & $\begin{array}{r}\text { Date } \\
\mathrm{dd} / \mathrm{mm} / \mathrm{yy}\end{array}$ & $\begin{array}{l}\lambda \text { Range } \\
{[\AA]}\end{array}$ & $\begin{array}{r}t_{\exp } \\
{[\mathrm{min}]}\end{array}$ & Remarks \\
\hline 1983.3 & IUE SWP & 19905 & $05 / 05 / 83$ & $1152-1978$ & 183 & \\
\hline \multirow[t]{2}{*}{1986.3} & IUE SWP & 28211 & $23 / 04 / 86$ & $1152-1978$ & 45 & \multirow[t]{2}{*}{ underexposed } \\
\hline & IUE LWP & 08083 & $23 / 04 / 86$ & $1853-3347$ & 240 & \\
\hline 1990.6 & CTIO 4m (a) & & $12 / 08 / 90$ & $3447-5096$ & 8 & $1.6^{\prime \prime}$ slit; $\times 1.3$ assumed \\
\hline \multirow[t]{2}{*}{1993.9} & IUE SWP & 49112 & $05 / 11 / 93$ & $1152-1978$ & 180 & \multirow{2}{*}{$\begin{array}{l}\text { SWP49075: no useful data } \\
1.33^{\prime \prime} \text { slit; } \times 1.3 \text { to match IUE }\end{array}$} \\
\hline & CTIO 4m (b) & & 28/01/94 & $3130-7410$ & 2 & \\
\hline \multirow[t]{5}{*}{1994.7} & CTIO 4m (b) & & $02 / 08 / 94$ & $3108-7375$ & 10 & $2^{\prime \prime}$ slit, $40 \%$ flux lost, not used \\
\hline & CTIO 4m (b) & & $02 / 08 / 94$ & $3108-7375$ & 5 & $10^{\prime \prime}$ slit, $\times 1.5$ to match IUE \\
\hline & IUE SWP & 52212 & $25 / 09 / 94$ & $1152-1978$ & 180 & saturated: not used \\
\hline & IUE SWP & 52222 & 26/09/94 & $1152-1978$ & 60 & \multirow{2}{*}{ \} weighted mean used } \\
\hline & IUE SWP & 52223 & 27/09/94 & $1152-1978$ & 90 & \\
\hline \multirow[t]{4}{*}{1995.0} & CTIO 4m (c) & & $31 / 12 / 94$ & $3184-7463$ & 10 & $2^{\prime \prime}$ slit; not used (40\% flux lost) \\
\hline & CTIO 4m (c) & & $31 / 12 / 94$ & $3184-7463$ & 5 & $10^{\prime \prime}$ slit; $\times 1.2$ to match IUE \\
\hline & IUE SWP & 53255 & 02/01/95 & $1152-1978$ & 60 & \multirow{2}{*}{\} weighted mean used } \\
\hline & IUE SWP & 53256 & $02 / 01 / 95$ & $1152-1978$ & 90 & \\
\hline 1995.3 & IUE SWP & 54312 & $06 / 04 / 95$ & $1152-1978$ & 180 & exposure segmented $2 \times 90 \mathrm{~min}(\mathrm{~g})$ \\
\hline \multirow[t]{2}{*}{1995.7} & CTIO 4m (d) & & $24 / 08 / 95$ & $3350-6737$ & 10 & \multirow{2}{*}{$\begin{array}{l}1.5^{\prime \prime} \text { slit, } \times 1.9 \text { to match IUE } \\
\text { weakly exposed }\end{array}$} \\
\hline & IUE SWP & 55790 & $01 / 09 / 95$ & $1152-1978$ & 90 & \\
\hline \multirow[t]{2}{*}{1995.9} & IUE SWP & 56155 & $06 / 11 / 95$ & $1152-1978$ & 120 & \multirow[b]{2}{*}{$2^{\prime \prime}$ slit, $\times 1.3$ to match IUE } \\
\hline & CTIO $1.5 \mathrm{~m}(\mathrm{e})$ & & $16 / 11 / 95$ & $3096-6534$ & 15 & \\
\hline \multirow[t]{6}{*}{1996.0} & HST FOS G130H & Y31L0105T & $11 / 12 / 95$ & $1087-1605$ & 190 & \multirow{6}{*}{ flux $15 \%$ lower than next obs.; not used } \\
\hline & HST FOS G190H & Y31L0106T & $11 / 12 / 95$ & $1573-2330$ & 43 & \\
\hline & HST FOS G400H & Y31L0107T & $11 / 12 / 95$ & $3239-4821$ & 35 & \\
\hline & CTIO-4m (d) & & $22 / 01 / 96$ & $3633-7330$ & 10 & \\
\hline & CTIO-4m (d) & & 24/01/96 & $3633-7330$ & 10 & \\
\hline & IUE SWP & 56787 & $10 / 02 / 96$ & 1152-1978 & 120 & \\
\hline \multirow[t]{6}{*}{1996.9} & HST FOS G130H & Y3J30106T & $12 / 11 / 96$ & $1087-1605$ & 63.2 & \multirow{6}{*}{\} mean used } \\
\hline & HST FOS G130H & Y3J30107T & $12 / 11 / 96$ & $1087-1605$ & 63.2 & \\
\hline & HST FOS G130H & Y3J30108T & $13 / 11 / 96$ & $1087-1605$ & 63.2 & \\
\hline & HST FOS G190H & Y3J30109T & $13 / 11 / 96$ & $1573-2330$ & 43 & \\
\hline & HST FOS G400H & Y3J3010AT & $13 / 11 / 96$ & $3239-4821$ & 15 & \\
\hline & CTIO 4m (d) & & $13 / 01 / 97$ & $3680-7390$ & 30 & \\
\hline \multirow[t]{4}{*}{1999.0} & HST STIS G140L & o55r01010 & $08 / 01 / 99$ & $1124-1730$ & 75 & FUV-MAMA \\
\hline & HST STIS G230L & o55r01020 & 08/01/99 & $1580-3160$ & 48 & NUV-MAMA \\
\hline & HST STIS G430L & o55r01030 & 08/01/99 & $2900-5710$ & 28 & $\mathrm{CCD}$ \\
\hline & HST STIS G750L & o55r02020 & 08/01/99 & 5236-10266 & 38 & $\mathrm{CCD}$ \\
\hline \multirow[t]{4}{*}{2000.1} & HST STIS G140L & o5im01010 & $04 / 02 / 00$ & $1120-1718$ & 100 & \multirow{4}{*}{$\begin{array}{l}\text { FUV-MAMA } \\
\text { NUV-MAMA } \\
\text { CCD }\end{array}$} \\
\hline & HST STIS G230L & o5im01020 & $04 / 02 / 00$ & $1580-3165$ & 61 & \\
\hline & HST STIS G430L & o5im02010 & $04 / 02 / 00$ & $2900-5712$ & 34 & \\
\hline & ESO 3.6-m (f) & & $05 / 03 / 00$ & $3229-8063$ & 30 & \\
\hline
\end{tabular}

(a) CTIO 4m, RC spectrograph, grating KPGL2, CCD detector.

(b) CTIO 4m, RC spectrograph, grating KPGL2, Ret1 detector.

(c) CTIO 4m, RC spectrograph, grating KPGL2, Ret2 detector.

(d) CTIO 4m, RC spectrograph, grating KPGL3, Loreal3K-1 detector.

(e) CTIO $1.5 \mathrm{~m}, \mathrm{RC}$ spectrograph, Loral1 $\mathrm{K}-1$ detector.

(f) ESO 3.6-m with EFOSC/2.9.

(g) INES and NEWSIPS IUE archives erroneously assume one single 90 min exposure and hence give wrongly calibrated data.

weeks are grouped together and assigned to a single "epoch". The overall variation of the UV flux can be seen from Fig. 1.

The ground-based spectra of our dataset are also photometrically calibrated. However, depending on the slit width and the seeing conditions, part of the stellar flux might have been lost. Therefore we compare each optical spectrum with the UV observations of the same epoch; if they do not fit together continuously, we derive an empirical correction factor to the optical fluxes. It turns out that most of the CTIO spectra have to be multiplied by factors of 1.3 , typically (see Table 1 ). The calibration problem seems to depend on the chosen slit width, as especially those spectra taken with small slit fall short - in some cases very obvious when we can compare with a spectrum taken in the same night but with a wider slit. At least, those apparent differences in flux level give not enough evidence for claiming a strong short-term variability of the object. 
Table 2. Relative line intensities of strong nebular UV lines, as measured from HST STIS long-slit spectra at off-star positions (southern nebular filaments). These values are used for correcting the IUE spectra for nebular contamination.

\begin{tabular}{lrr}
\hline \hline N IV & 1240.13 & 264 \\
Si IV & 1393.76 & 92 \\
Si IV & 1402.77 & 46 \\
N IV & 1483.33 & 360 \\
C IV & 1548.19 & 260 \\
C IV & 1550.76 & 130 \\
He II & 1640.43 & 1000 \\
\hline
\end{tabular}

\subsection{Radial velocity}

The heliocentric radial velocity of the system was determined as $+300 \mathrm{~km} \mathrm{~s}^{-1}$ by Peña et al. (2003) from the nebular lines, using ESO VLT UVES spectra. High-resolution long-slit HST STIS spectra, obtained during the same observing runs as the STIS spectra applied in the preset study (epochs 1999.0 and 2000.1), confirm that value for the radial velocity of the central star. Therefore we shift the wavelength scale of the observed spectra correspondingly before fitting them with theoretical spectra. The match in wavelength is always excellent, never showing any systematic offset. However, the stellar lines are broad and therefore small shifts (below $\approx 50 \mathrm{~km} \mathrm{~s}^{-1}$ ) would escape from detection.

\subsection{Nebular subtraction}

Both the IUE UV and the ground-based visual observations show a strong nebular contamination superimposed to the stellar spectrum. The nebula of N 66 falls entirely into the "Large Aperture" of the IUE spectrograph. Only the high spatial resolution of the HST allows to isolate the stellar spectrum alone, but even these data are still contaminated by the strongest of the nebular lines ([O III] 5007, $\mathrm{H} \alpha, \mathrm{H} \beta$ ).

In particular, from the times before the brightness outburst there exists only one well-exposed UV spectrum (epoch 1983.3), and this IUE spectrum is strongly nebular contaminated because the star was still faint at that time. Because of the strategic importance of the few emission features visible in these data, we try carefully to separate their nebular and stellar contribution, respectively. The next well-exposed IUE SWP spectrum available is from epoch 1994.7, when the star was already much brighter and the nebular contribution to the strategic lines became unimportant.

\subsubsection{UV lines}

From our HST STIS long-slit spectra (see below) we measured the relative intensities of nebular lines at off-star positions (Table 2). These values are used to correct the IUE spectra for nebular contamination. A synthetic "nebular line spectrum" is constructed assuming Gaussians of $5 \AA$ FWHM (tentatively the instrumental profile). The flux of the He II 1640 nebular line is used as reference (relative line intensity $=1000$ ). However, the observed line is the sum of the stellar and nebular contribution.
We adjust the absolute flux of the nebular He II 1640 line such that the semi-forbidden N IV line at $1483 \AA$ vanishes (but note that the blending N IV 1486 line is stellar). A plausible result is achieved when a flux of $4 \times 10^{-13} \mathrm{erg} \mathrm{cm}^{-2} \mathrm{~s}^{-1}$ is adopted for the nebular He II 1640 line. In the pre-outburst IUE spectrum from epoch 1983.3, this leaves $3 \times 10^{-13} \mathrm{erg} \mathrm{cm}^{-2} \mathrm{~s}^{-1}$ for the stellar flux in that line. This compares well with the flux of $2 \times 10^{-13} \mathrm{erg} \mathrm{cm}^{-2} \mathrm{~s}^{-1}$ measured in the latest post-outburst (epoch 2000.1) HST spectrum which is essentially free of nebular contamination.

\subsubsection{UV continuum}

The total nebular He II 1640 line flux of $4 \times 10^{-13} \mathrm{erg} \mathrm{cm}^{-2} \mathrm{~s}^{-1}$ is also employed for correcting the UV continuum. For that purpose, a nebular model is calculated and scaled to that He II 1640 line flux. Then the theoretical nebular continuum is reddened and subtracted from the IUE observations.

The nebular continuum calculation accounts for recombination of $\mathrm{H}^{+}, \mathrm{He}^{+}$and $\mathrm{He}^{++}$, and for two-photon emission. The ionic abundances for $\mathrm{He}^{+}$and $\mathrm{He}^{++}$were adopted from Peña et al. (1995). The emission coefficients were taken from Aller (1984) at an electron temperature of $16 \mathrm{kK}$ as appropriate for the ionized gas in N 66 .

\subsubsection{Optical continuum}

Similarly to the UV, a (reddened) theoretical nebular continuum is subtracted from the (absolutely calibrated) groundbased visual spectra. In the optical, the models are scaled in relation to $\mathrm{H} \beta$. As the observed $\mathrm{H} \beta$ line is composed by a stellar and a nebular contribution, only the narrow nebular emission is measured for that purpose. In some of our observations (i.e. those with $10^{\prime \prime}$ wide slit) the spectral resolution is not sufficient to isolate the nebular component; in those cases we measure the total line and subtract the stellar contribution according to our models. There are indicators (Balmer jump, spectral slope) which prove that the whole procedure of subtracting a theoretical nebular continuum works fine, but sometimes does a bit of over-correction for some unknown reason. In those cases we reduce the correction arbitrarily by about $20 \%$.

\section{The models}

A "standard" WR atmosphere is assumed to be sphericallysymmetric, homogeneous and stationary. The usual $\beta$-law with $\beta=1$ is adopted for the expansion velocity. The "stellar radius" $R_{*}$, which is the inner boundary of our model atmosphere, corresponds per definition to a Rosseland optical depth of 20. The "stellar temperature" $T_{*}$ is defined as the effective temperature referred to that radius $R_{*}$.

Wind inhomogeneities ("clumping") are accounted for in a first-order approximation (cf. Hamann \& Koesterke 1998b). The clumps are assumed to fill a volume fraction $f_{\mathrm{V}}=D^{-1}$ while the interclump space is void.

Any particular WR atmosphere thus is specified by its basic parameters $T_{*}, R_{*}, \dot{M}, v_{\infty}$ and chemical composition (given e.g. as mass fractions $X_{\mathrm{He}}, X_{\mathrm{N}}$ etc.). 
Schmutz et al. (1989) discovered an approximate degeneracy in this parameter space, which in its later version (Hamann \& Koesterke 1998b) additionally incorporates the clumping contrast $D$ for inhomogeneous winds. Defining a socalled transformed radius $R_{\mathrm{t}}$

$R_{\mathrm{t}}=R_{*}\left[\frac{v_{\infty}}{2500 \mathrm{~km} \mathrm{~s}^{-1}} \mid \frac{\dot{M} \sqrt{D}}{10^{-4} M_{\odot} \mathrm{yr}^{-1}}\right]^{2 / 3}$,

it turns out that models with same $R_{\mathrm{t}}$ exhibit almost the same emission line equivalent widths, irrespective of different combinations of $\dot{M}, R_{*}$ and $v_{\infty}$ (while, of course, $T_{*}$, composition etc. are fixed). This (approximate) invariance, which was validated by various numerical experiments with reasonable accuracy, will be exploited to facilitate our spectral analyses presented below.

The synthetic spectra for our analyses are calculated with the Potsdam code for expanding stellar atmospheres. In this code, the radiation transfer is formulated in the co-moving frame of reference (CMF). Doppler broadening by "microturbulence" is generally set to $100 \mathrm{~km} \mathrm{~s}^{-1}$. The statistical equations balance all relevant radiative and collisional transition rates. The consistent solution of radiative transfer and statistical equilibrium is achieved by means of an Accelerated Lambda Iteration (ALI) technique (e.g. Hamann 2003). A recent improvement of WR model atmospheres concerns the (approximate) inclusion of iron line blanketing by means of the "superlevel" concept (cf. Gräfener et al. 2002).

The temperature stratification is obtained from the constraint of radiative equilibrium. It is established by a temperature correction scheme generalized from the ideas of Unsöld and Lucy, and accelerated by an ALI technique as well (Hamann \& Gräfener, in preparation).

After the non-LTE population numbers have been obtained, the atomic data are further refined by splitting the levels and multiplets in the observed spectral range as far as necessary, and the emergent spectra are calculated in the observer's frame ("formal integral"). Frequency redistribution by electron scattering is taken into account in this step. - More details of our model calculations are given in Gräfener et al. (2002) and references therein.

Our N 66 models account for the elements $\mathrm{H}, \mathrm{He}, \mathrm{C}, \mathrm{N}$ and Fe. The model atoms (cf. Table 3) comprise 196 energy levels with 1672 line transitions in Non-LTE (among those, 742 lines with very low $f$-value are treated in an approximate way). Low-temperature dielectronic recombination to N IV is accounted for by 464 stabilizing transitions from auto-ionizing states. Note that due to the high effective temperature of $\mathrm{N} 66$ we could neglect low ionization stages like C III and N III. For Fe, 72 energy levels with 360 line transitions are in fact "superlevels" and "superlines" comprising $10^{4}$ individual levels and $10^{7}$ lines of the iron group elements in order to account for "iron line blanketing" (cf. Gräfener et al. 2002).

\section{The analyses}

The model spectra described in the previous section are compared with the observations of $\mathrm{N} 66$ from different epochs listed
Table 3. Model atom summary: number of Non-LTE energy levels and line transitions.

\begin{tabular}{lrr}
\hline \hline Ion & Levels & Lines \\
\hline H I & 10 & 45 \\
H II & 1 & 0 \\
He I & 17 & 136 \\
He II & 16 & 120 \\
He III & 1 & 0 \\
C III & 2 & 1 \\
C IV & 19 & 171 \\
C V & 1 & 0 \\
N III & 1 & 0 \\
N IV & 38 & 703 \\
N V & 17 & 136 \\
N VI & 1 & 0 \\
Fe III & 1 & 0 \\
Fe IV & 11 & 49 \\
Fe V & 13 & 69 \\
Fe VI & 17 & 121 \\
Fe VII & 11 & 52 \\
Fe IIX & 9 & 34 \\
Fe IX & 9 & 35 \\
Fe X & 1 & 0 \\
\hline Sum & 196 & 1672 \\
\hline & &
\end{tabular}

in Table 1. Spectral fits are plotted for each of the epochs; only two examples can be shown here (Figs. 2 and 3) for brevity. The various model parameters are manually adjusted such that the fit is optimized. This is clearly an iterative procedure; fortunately, the influence of the individual parameters on individual properties of the spectrum is sufficiently decoupled and linear to allow for their unique determination.

It is reasonable to assume that distance, reddening and chemical composition are the same for all epochs, while other parameters (mass-loss rate, luminosity, stellar temperature, radius, wind velocity) are potentially variable.

\subsection{Time-independent parameters: Reddening and distance}

In our fit procedure, we compare the observed (nebulacorrected) spectrum with the synthetic stellar spectra, after the latter was reddened due to interstellar absorption. Best suited for determining the color excess are the HST spectra from 1999 with their broad wavelength coverage. We apply the extinction law by Seaton (1979) for the Galactic foreground with $E_{B-V}^{\mathrm{Gal}}=0.03 \mathrm{mag}$, and the law by Howarth (1983) for the LMC. The color excess of the LMC contribution is determined to $E_{B-V}^{\mathrm{LMC}}=0.13 \mathrm{mag}$ from a careful comparison between observed and calculated flux distributions. Clearly, as the latter depends somewhat on the model parameters, this is an iterative procedure. The obtained total color excess of $0.16 \mathrm{mag}$ lies within the range of 0.10 to 0.18 mag derived from the Balmer decrement of the nebular lines (Peña et al. 1995, 2003). The applied LMC reddening law predicts a broad absorption 
W.-R. Hamann et al.: The central star of LMC-N 66: Its dramatic evolution 1983-2000
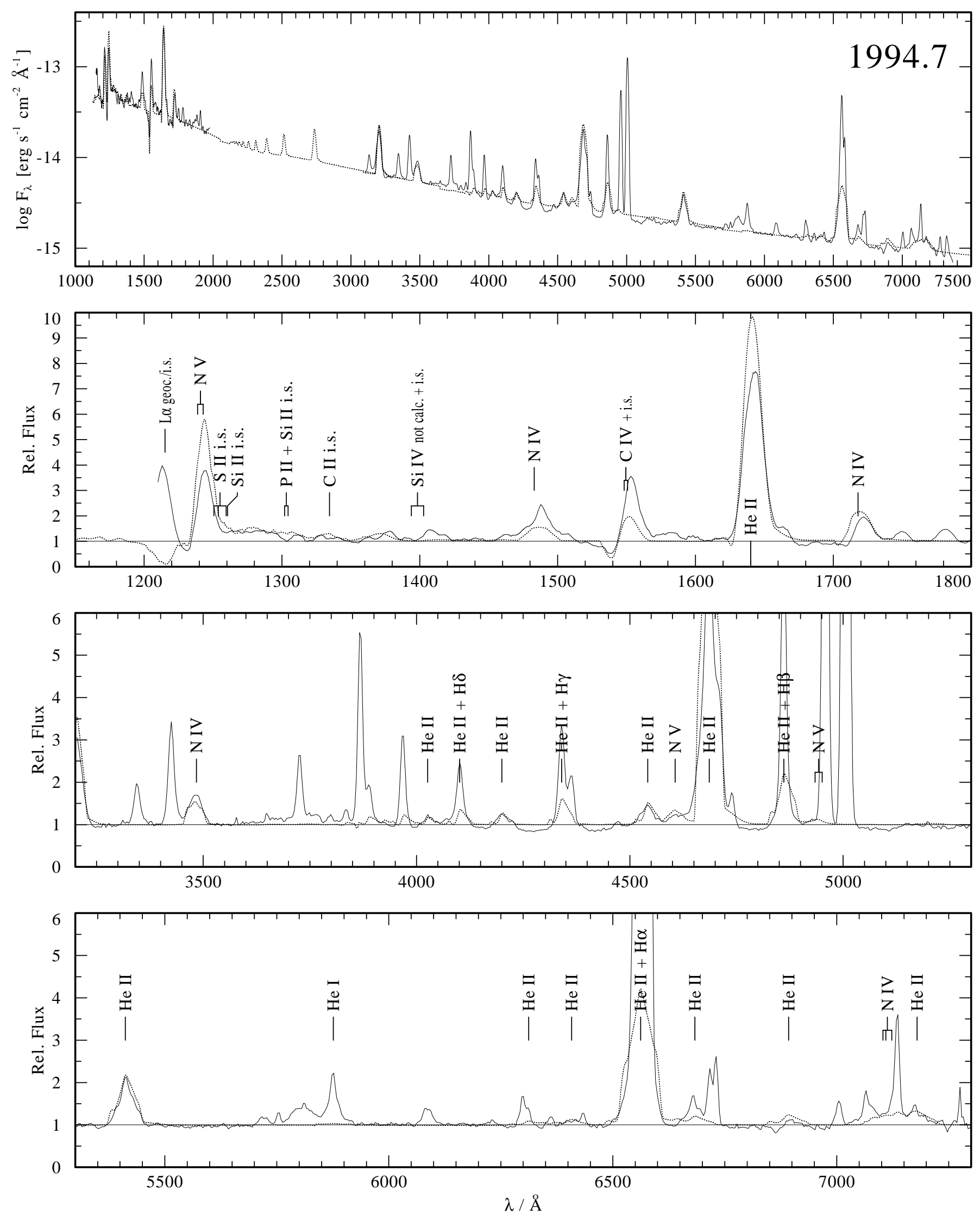

Fig. 2. Spectrum of N 66 (continuous line) at epoch 1994.7, i.e. when the star was brightest, observed with IUE (UV) and CTIO (visual), after subtracting nebular continuum and nebular UV lines (see text). Superimposed is the synthetic model B scaled to a luminosity of $\log L / L_{\odot}=5.43$ (dotted line). While the uppermost panel shows absolute fluxes, in the lower three panels the observed fluxes were divided by the model continuum. 

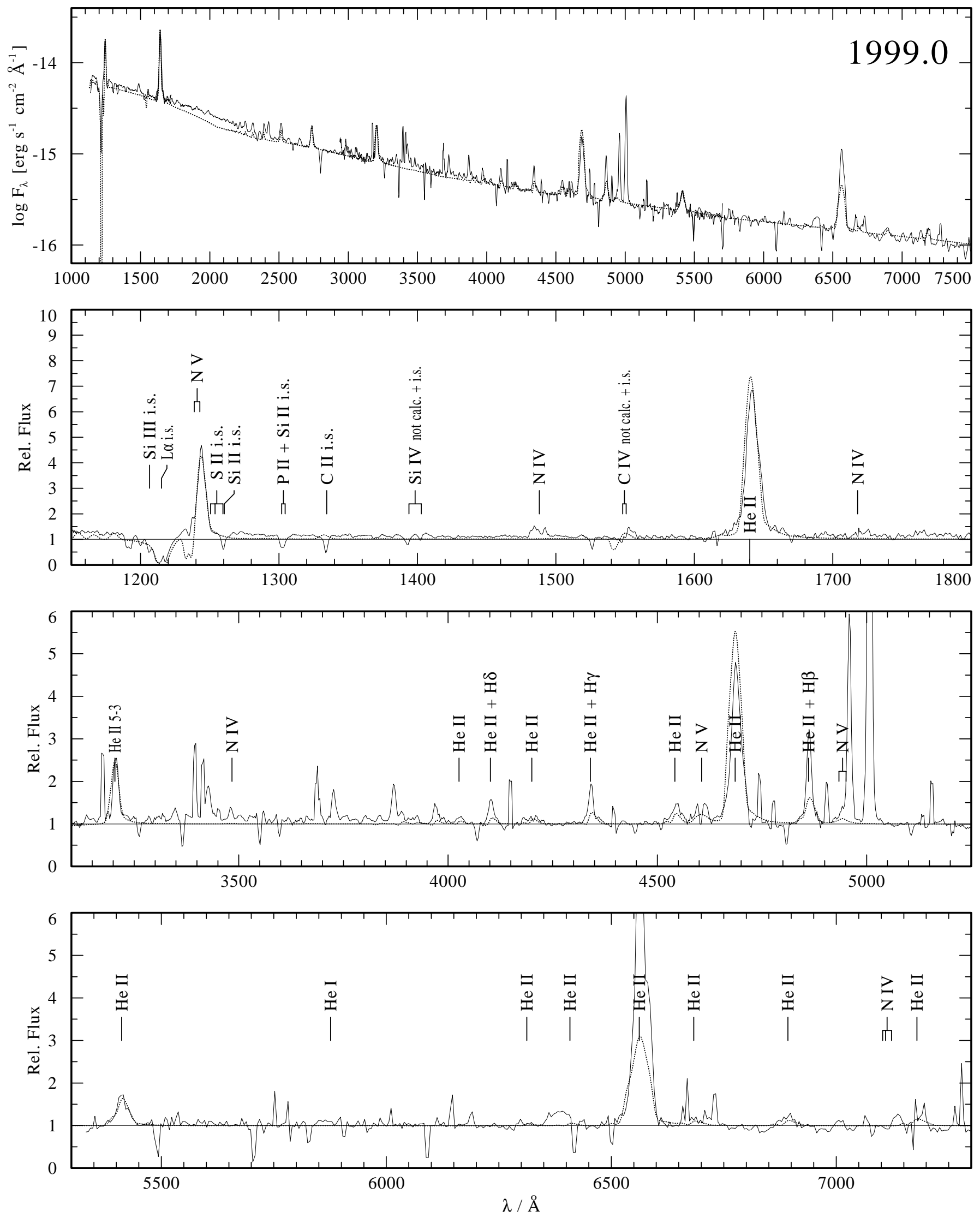

Fig. 3. Spectrum of N 66 (continuous line) at epoch 1999.0, i.e. when the star was back to low brightness, observed with the HST. Superimposed is the synthetic model C scaled to a luminosity of $\log L / L_{\odot}=4.58$ (dotted line). While the uppermost panel shows absolute fluxes, in the lower three panels the observed fluxes were divided by the model continuum. 


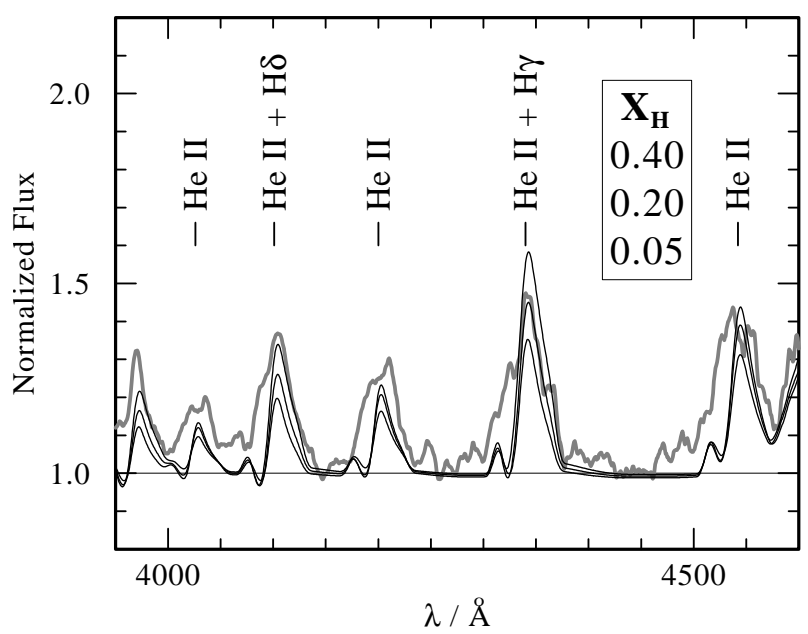

Fig. 4. HST observation (grey thick line) of epoch 1996.0 compared with three models (black thin lines) of different hydrogen abundances (mass fractions $X_{\mathrm{H}}=0.40,0.20$ and 0.05 , respectively). Along the He II Pickering line series, every other member is blended with a $\mathrm{H}$ Balmer line and therefore stronger, compared to the monotonic decrement expected from hydrogen-free atmosphere. The model with $X_{\mathrm{H}}=0.20$ roughly reproduces the observed up-and-down, while hydrogen mass fractions as high as $40 \%$ or as low as $5 \%$ can be ruled out.

feature around $2100 \AA$ which is not present in our observations (cf. Fig. 3, uppermost panel). Obviously this average law does not conform exactly with the conditions along the specific line-of-sight towards N 66. Interstellar Lyman-alpha absorption is modeled with a column density of $3.8 \times 10^{21} \mathrm{~cm}^{-2}$ per one magnitude of $E_{B-V}$ (Groenewegen \& Lamers 1989). The model fluxes are scaled to the distance of the LMC, adopting a distance modulus of $18.45 \mathrm{mag}$ (Feast 1988). The most recent Cepheid calibration nearly confirmed that value (18.50 $\pm 0.15 \mathrm{mag}$, Benedict et al. 2002).

\subsection{Time-independent parameters: Chemical composition}

At all epochs, the spectrum of $\mathrm{N} 66$ looks similar to that of a massive Wolf-Rayet star of the nitrogen sequence with early subtype. The dominance of helium and nitrogen lines indicates that its atmosphere is composed of CNO-processed material, i.e. mainly of helium with traces of nitrogen while carbon and oxygen are underabundant.

However, WN atmospheres may contain a rest of hydrogen from incomplete CNO burning. This is not easily detected, because all $\mathrm{HI}$ lines are blended (within the broad line profiles from a rapidly expanding atmosphere) with He II. Even worse in our case, nebular emission further masks stellar hydrogen.

In Paper I we stated that N66 "shows an extremely H-deficient atmosphere, with $X / Y \leq 0.1$ ". Now we carefully re-examine the hydrogen abundance. Most suitable for that purpose are our HST FOS spectra (e.g. from epoch 1996.0), because they most effectively suppress the nebular emission. In fact we now find that along the He II Pickering series every second line (with even principle quantum numbers, i.e. coinciding with a H I Balmer line) is outstandingly strong, clearly

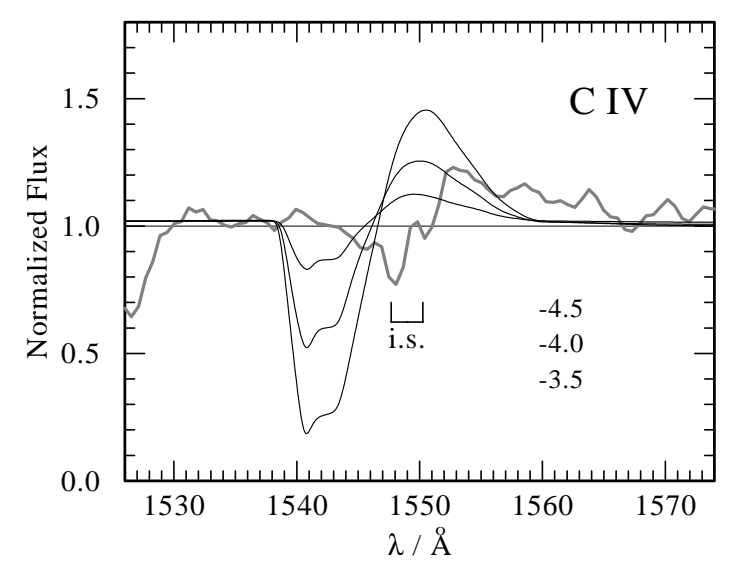

Fig. 5. HST observation (grey thick line) of epoch 2000.1 compared with three models (black thin lines) of different carbon abundances (logarithm of the mass fraction $\log \left(X_{\mathrm{C}}\right)=-4.5,-4.0$ and -3.5 , respectively. Narrow interstellar absorptions are superimposed on a weak stellar PCygni profile. The model with $\log X_{\mathrm{C}}=-4.0$ predicts an emission of about the observed strength, but a much stronger absorption component than observed.

indicating some stellar hydrogen. Quantitative fits yield a hydrogen mass fraction of $0.2 \pm 0.1$, roughly (cf. Fig. 4). Although the detailed fit is not very good, hydrogen abundances higher than 0.4 and smaller than 0.05 (by mass) can be excluded.

For the nitrogen abundance we assume $0.3 \%$ per mass. This reproduces the observed balance between the strengths of the He II lines (especially He II 1640) and the N V resonance line, but due to the parameter sensitivity of both lines this is rather a plausible assumption than an empirical determination. Given that in CNO-processed material almost all initial $\mathrm{CNO}$ has been converted into $\mathrm{N}$, the assumed $\mathrm{N}$ abundance corresponds to $\frac{1}{4}$ of solar metallicity.

Small traces of carbon are indicated by the C IV 1548/1550 resonance doublet. From a series of test models (cf. Fig. 5) we estimate that the mass fraction of carbon is definitely smaller than $\log X_{\mathrm{C}}=-4.0$, which corresponds to less than $\frac{1}{40}$ solar.

An iron mass fraction of $X_{\mathrm{Fe}}=2 \times 10^{-4}$ yields a good fit to the iron forest between 1260 and $1400 \AA$ which is nicely visible at those epochs with densest wind (e.g. 1995.3 and model B). Given the parameter dependence, this value is also to be taken with caution. It corresponds roughly to $\frac{1}{7}$ of the solar value. Those parameters of our final models which are kept constant for all epochs are compiled in Table 4.

\subsection{The dramatic time variability}

An inspection of the available time series of spectra from the central star of $\mathrm{N} 66$ reveals two basic facts:

1. The star increased its brightness after 1991 dramatically by almost one order of magnitude, reaching the maximum in 1994/1995, and returned to its previous low state after about four more years in 1999 .

2. The stellar spectrum, always of Wolf-Rayet (WN) type, remains amazingly similar over the whole event, despite the scaling of the absolute flux level. This constancy holds for the (normalized) line spectrum as well as for the gross 
Table 4. Parameters which do not vary with the epoch.

\begin{tabular}{ll}
\hline \hline$T_{\star}$ & $112 \mathrm{kK}$ \\
$D$ & 10 \\
$v_{\mathrm{D}}$ & $100 \mathrm{~km} \mathrm{~s}^{-1}$ \\
$X_{\mathrm{H}}$ & 0.2 \\
$X_{\mathrm{N}}$ & $310^{-3}$ \\
$X_{\mathrm{C}}$ & $\lesssim 1 \times 10^{-4}$ \\
$X_{\mathrm{Fe}}$ & $2 \times 10^{-4}$ \\
\hline$M-m$ & $18.45 \mathrm{mag}$ \\
$E_{B-V}(\mathrm{Gal})$. & $0.03 \mathrm{mag}$ \\
$E_{B-V}(\mathrm{LMC})$ & $0.13 \mathrm{mag}$ \\
\hline
\end{tabular}

shape of the spectral energy distribution from the UV to red-optical wavelengths.

In Paper I we restricted ourselves to model only the increase of continuum brightness. As we have shown, this can be done by assuming a mass-loss outburst. For simplicity, all other parameters including the luminosity were kept constant. The continuum brightness grows with higher mass-loss rate, because the pseudo-photosphere (i.e. radius of optical depths unity) is inflated while the emergent spectral energy redistribution is shifted to longer wavelengths, from the extreme UV to the UV and visual.

However, this simplified interpretation cannot be retained when we now include the line spectra into our analysis. The (approximate) constancy of the line spectrum implies that the stellar temperature and the transformed radius $R_{\mathrm{t}}$ (cf. Eq. (1)) remain roughly the same. (Recall that WR models which agree in $T_{*}$ and $R_{\mathrm{t}}$ yield nearly identical spectra, despite of a scaling with the square of the stellar radius, $R_{*}^{2}$.) Hence the observed brightness increase in the visual and UV during the outburst is accompanied by a corresponding increase of the bolometric stellar luminosity.

For restricting the model parameters in the $\log T_{*}-\log R_{\mathrm{t}^{-}}$ plane, we can employ the lines of N IV, N V and He II. Our published grid of WN models (Hamann \& Koesterke 1998a) provides a first orientation. Note, however, that the more advanced, line-blanketed models applied in the present paper give modified results.

The clear appearance of several N IV lines especially in the spectra at outburst (cf. Fig. 2) restrict the parameters to a very narrow strip in the $\log T_{*}-\log R_{\mathrm{t}}$-plane; hotter or thinner winds are more ionized and do not show any NIV, while cooler or thicker winds give huge N IV lines.

Outside outburst, the N IV lines are only marginally detectable (cf. Fig. 3). But now the observed N V lines come into play. Those NV lines only appear in the models for some temperature range around $100 \mathrm{kK}$ (cf. Hamann \& Koesterke 1998a). Therefore we can exclude that the stellar temperature of N 66 changed drastically between outburst and quiet state.

Reasonable fits of our observed spectra from all epochs can be obtained with the same stellar temperature $T_{*}=112 \mathrm{kK}$ $\left(\log T_{*} / \mathrm{K}=5.05\right)$, with an uncertainty of $\pm 20 \mathrm{kK}$ in the worst case. The observed variability in the line spectrum requires
Table 5. Parameters of N 66 obtained by model fits to the observed spectra from the different epochs. $\Delta(\log L)$ is the logarithm of the luminosity, relative to $\log L / L_{\odot}=4.78$. Apart from the luminosity scaling, only three different models (A, B, C) are applied. The mass-loss rate is in the usual units, $M_{\odot} \mathrm{yr}^{-1}$.

\begin{tabular}{ccccccc}
\hline \hline Epoch & $\log \frac{R_{\mathrm{t}}}{R_{\odot}}$ & $v_{\infty} / \frac{\mathrm{km}}{\mathrm{s}}$ & Model & $\Delta \log L$ & $\frac{R_{*}}{R_{\odot}}$ & $\log \dot{M}$ \\
\hline 1983.3 & 0.50 & 2200 & $\mathrm{~A}$ & -0.20 & 0.52 & -5.66 \\
1986.3 & 0.50 & 2200 & $\mathrm{~A}$ & -0.20 & 0.52 & -5.74 \\
1990.6 & 0.50 & 2200 & $\mathrm{~A}$ & -0.20 & 0.52 & -5.74 \\
1993.9 & 0.45 & 2200 & $\mathrm{~B}$ & 0.35 & 0.98 & -5.25 \\
1994.7 & 0.45 & 2200 & $\mathrm{~B}$ & 0.65 & 1.38 & -5.02 \\
1995.0 & 0.45 & 2200 & $\mathrm{~B}$ & 0.35 & 0.98 & -5.25 \\
1995.3 & 0.45 & 2200 & $\mathrm{~B}$ & 0.45 & 1.09 & -5.17 \\
1995.7 & 0.50 & 2200 & $\mathrm{~A}$ & 0.10 & 0.73 & -5.51 \\
1995.9 & 0.50 & 2200 & $\mathrm{~A}$ & 0.20 & 0.82 & -5.44 \\
1996.0 & 0.50 & 2200 & $\mathrm{~A}$ & 0.20 & 0.82 & -5.44 \\
1996.9 & 0.50 & 2200 & $\mathrm{~A}$ & 0.10 & 0.73 & -5.51 \\
1999.0 & 0.50 & 1600 & $\mathrm{C}$ & -0.20 & 0.52 & -5.67 \\
2000.1 & 0.50 & 1600 & $\mathrm{C}$ & -0.20 & 0.52 & -5.67 \\
\hline
\end{tabular}

Table 6. Some properties of the final models (cf. Table 5).

\begin{tabular}{lccc}
\hline \hline & \multicolumn{3}{c}{ Model } \\
& $\mathrm{A}$ & $\mathrm{B}$ & $\mathrm{C}$ \\
\hline $\log \left(R_{\mathrm{t}} / R_{\odot}\right)$ & 0.50 & 0.45 & 0.50 \\
$v_{\infty} /\left(\mathrm{km} \mathrm{s}^{-1}\right)$ & 2200 & 2200 & 1600 \\
$R(\tau=2 / 3) / R_{*}$ & 1.20 & 1.29 & 1.44 \\
$T_{\mathrm{eff}}(\tau=2 / 3) / \mathrm{kK}$ & 102 & 99 & 94 \\
$T_{\mathrm{e}}(\tau=2 / 3) / \mathrm{kK}$ & 88 & 85 & 85 \\
$T_{\text {Zanstra }}(\mathrm{He} \mathrm{II}) / \mathrm{kK}$ & 62 & 22 & 80 \\
\hline
\end{tabular}

only a tiny change in the transformed radius (i.e. the massloss rate), from $\log R_{\mathrm{t}} / R_{\odot}=0.50$ in the quiet state to 0.45 at outburst (see Table 5, models A and B).

Thus we must attribute the whole change in brightness to a change in the stellar radius, while $T_{*}$ and $R_{\mathrm{t}}$ remain fairly constant. Hence the mass-loss rate varies in parallel with the brightness outburst (see Fig. 6). We find $\dot{M} \propto L^{0.87}$ (Fig. 7), close to the exponent of 0.75 which would correspond to $R_{\mathrm{t}}=$ constant.

In the spectra during outburst, the width of the emission lines is well reproduced with a terminal wind velocity of $v_{\infty}=$ $2200 \mathrm{~km} \mathrm{~s}^{-1}$. Back to the quiet state the lines are a bit narrower and a model with $v_{\infty}=1600 \mathrm{~km} \mathrm{~s}^{-1}$ fits better (cf. Table 5, model $\mathrm{C}$ ). The lines might have been also that narrow before the outburst, but the low-resolution IUE data do not allow for a precise estimate.

Note that the "stellar temperature" $\left(T_{*}=112 \mathrm{kK}\right)$ is defined as the effective temperature, related to the "stellar radius" $R_{*}$ which we arbitrarily put at a Rosseland mean optical depth of 20 . The radius of the visible photosphere, $R(\tau=2 / 3)$, is larger (cf. Table 6). Correspondingly, the effective temperature $T_{\text {eff }}(\tau=2 / 3)$ referring to that radius is lower according to 


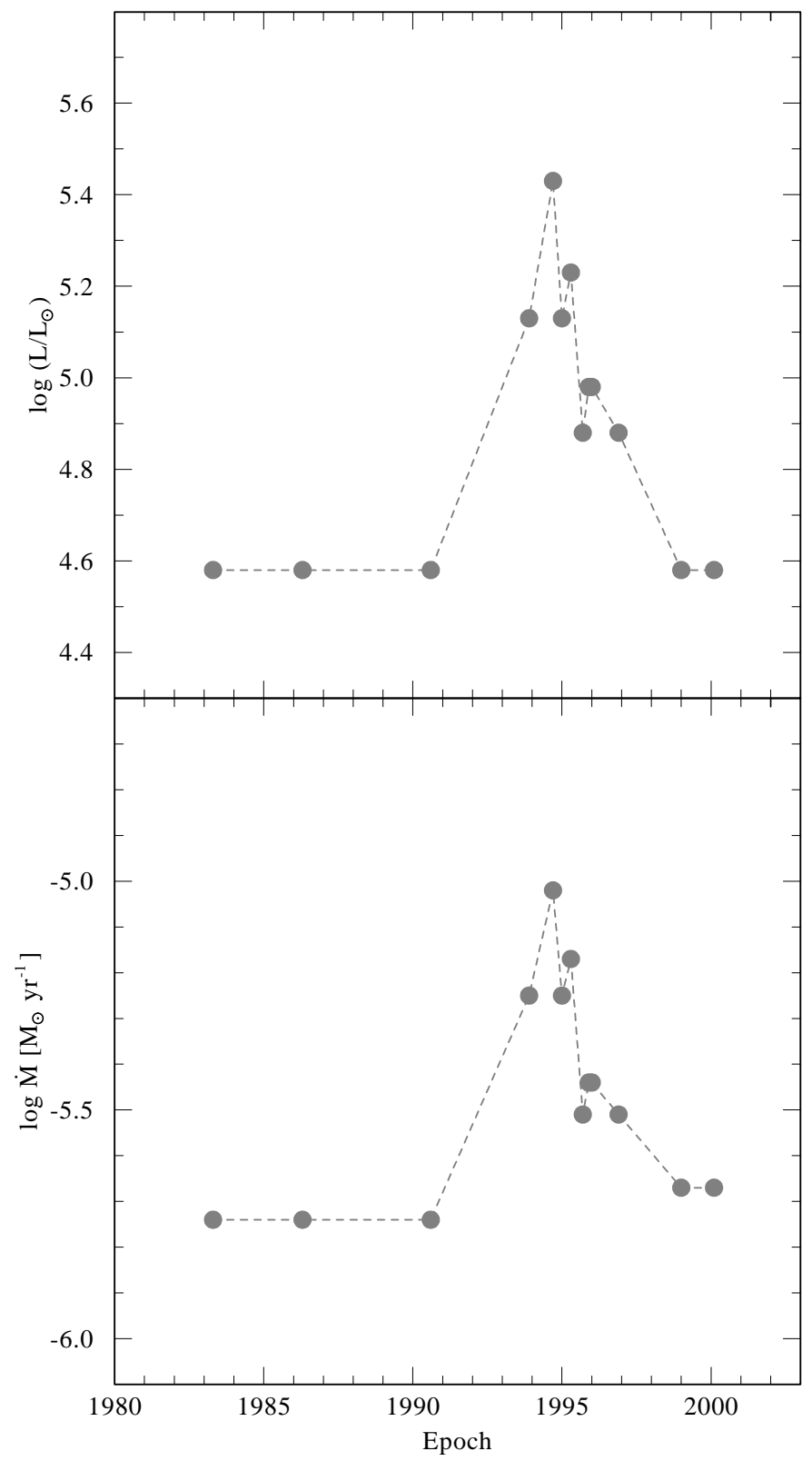

Fig. 6. Luminosity and mass-loss rate of N 66 as function of time.

Stefan-Boltzmann's law. The electron temperature $T_{\mathrm{e}}(\tau=2 / 3)$ at that photospheric radius is about $85 \mathrm{kK}$ for all three models.

Thus, apart from the drastically changing stellar radius and the corresponding scaling of $L$ and $\dot{M}$, the three "final models" $\mathrm{A}, \mathrm{B}$ and $\mathrm{C}$ differ only marginally. However, the slight reduction in the transformed radius, from $\log R_{\mathrm{t}} / R_{\odot}=0.50$ (models A, C) to 0.45 (model B), leads to recombination of He II at some distance from the star, due to the higher wind density. Consequently the resulting He II Zanstra temperatures differ drastically, being high $(62 \mathrm{kK}$ and $80 \mathrm{kK})$ for models A and C, respectively, but low $(22 \mathrm{kK})$ for the denser model B.

\section{The nature and origin of $\mathrm{N} 66$}

In the previous section we presented a detailed spectral analysis of the PN central star LMC-N 66 with respect to the stellar parameters and the atmospheric composition. Apart from the

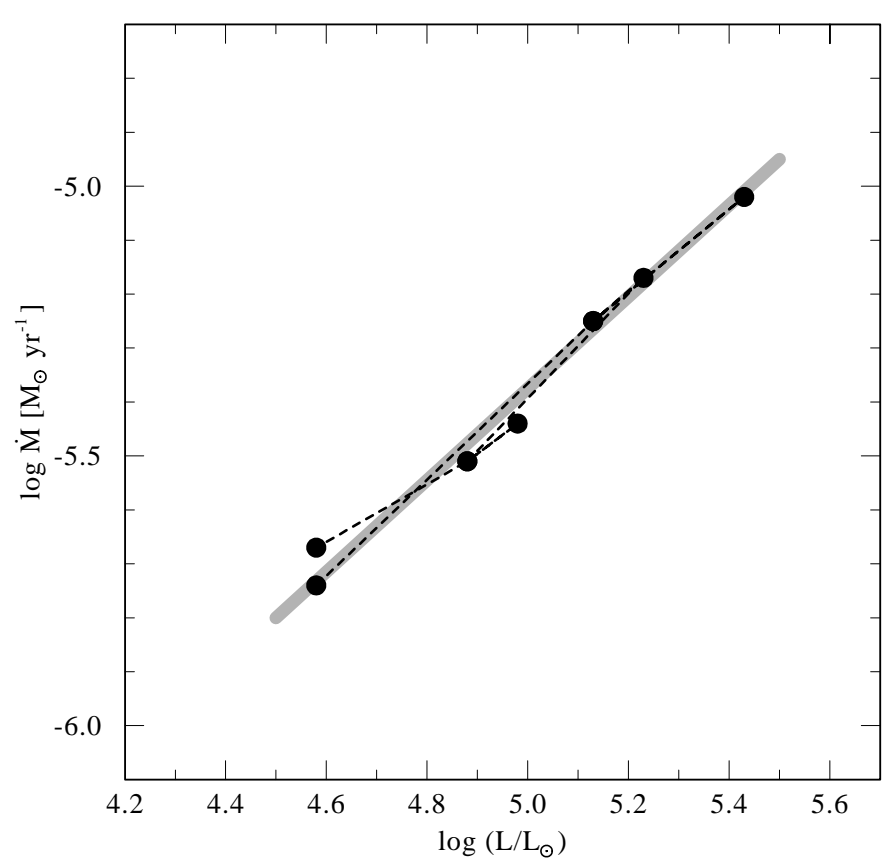

Fig. 7. Correlation between mass-loss rate and luminosity of N66 for the different epochs. The grey thick stripe indicates a slope of 0.87 .

dramatic variability of that star, already those parameters are quite unique and puzzling. Before discussing the nature and origin of N66, we summarize the basic facts which should be understandable in an acceptable scenario.

First, it should explain the location of N66 in the HR diagram, especially its luminosity. Second, the chemical composition (helium dominated, $20 \%$ hydrogen, carbon depletion) yields strong constraints. Third, there is a fast $\left(v_{\infty} \approx\right.$ $2000 \mathrm{~km} \mathrm{~s}^{-1}$ ) stellar wind. A mechanism for the dramatic brightness outburst is to be identified. The Helmholtz-Kelvin timescale of the involved part of the star must allow for the observed rapid luminosity change.

Furthermore, the existence and properties of the nebula around N 66 must fit into the picture. Kinematics and structure of this nebula have been studied by Dopita et al. (1985), Blades et al. (1992), and most recently by Peña et al. (2003). Its main body consists of two bright lobes oriented in opposite direction relative to the central star, which resemble a bipolar jet-like ejection. Their difference in radial velocity is larger than $80 \mathrm{~km} \mathrm{~s}^{-1}$. Several knots near the central star also show this bipolar kinematics. The kinematical age of the nebular features is of the order of 5000 years for the large loops, but it could be only 500 years for those knots closest to the star. A dark zone, separating the bright lobes, could have been produced by a dense torus around the central star which had impeded the expansion of the nebula in the equatorial plane. The total mass in the nebula amounts to about $0.6 M_{\odot}$ (Dopita et al. 1993). The chemical composition of the nebula is found to be normal (Paper I), i.e. dominated by hydrogen. 


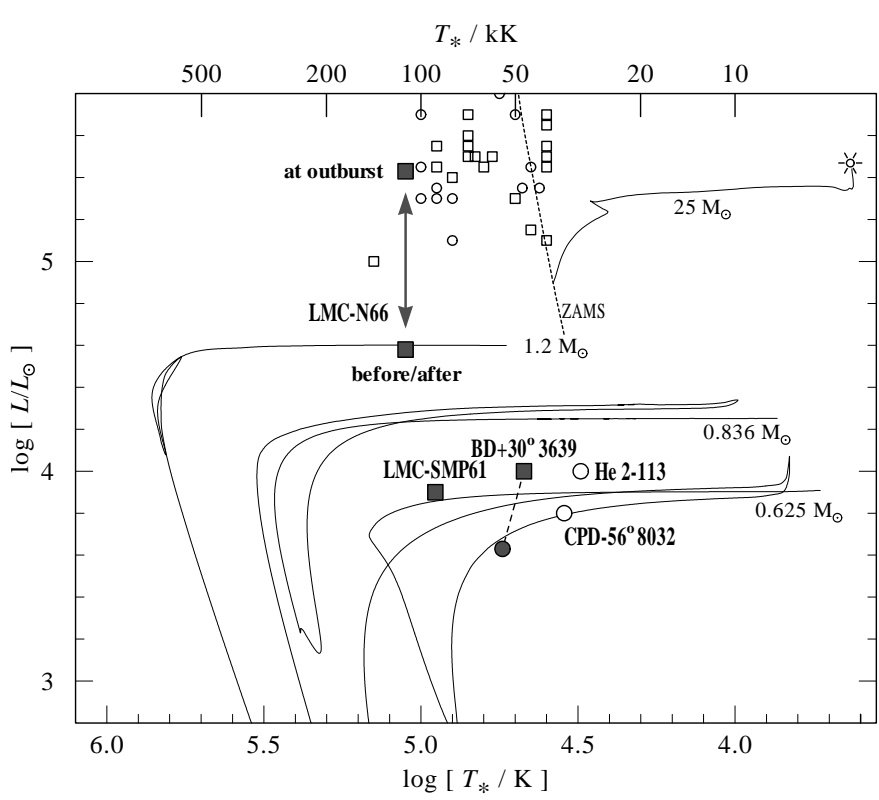

Fig. 8. Hertzsprung-Russell diagram with the path of $\mathrm{N} 66$ between quiet state and outburst. For comparison, some other hydrogendeficient (but [WC] type) central stars of well-determined luminosities based on known distances are given (full symbols). LMC-SMP 61 ( $d=50 \mathrm{kpc}$ from LMC membership) has been analyzed recently by Gräfener et al. (2003). The results for $\mathrm{BD}+30^{\circ} 3639(d=1.2 \mathrm{kpc}$ from the secular expansion of the nebula) by Leuenhagen et al. (1996) and Crowther et al. (2003) are represented by a filled square and dot, respectively. The distances of He 2-113 and CPD $-56^{\circ} 8032$ (open symbols, analyzed also by Leuenhagen et al. 1996) are from less reliable methods. Post-AGB evolutionary tracks (from Blöcker 1993, 1995; Paczyński 1970) are shown for rough comparison and reveal that [WC] type central stars have the same typical mass around $0.6 M_{\odot}$ as hydrogen-rich central stars. The outbursting [WN] star N66, however, is much more luminous. On the other hand, N66 is less luminous than any of the Galactic massive early-type WN stars (open symbols) analyzed by Hamann \& Koesterke (1998a). Standard evolutionary tracks for massive stars (as given for an initial mass of $25 M_{\odot}$, from Schaller et al. 1992) do not lead to WN stars at such luminosities, as they end in a supernova explosion as red supergiants.

\subsection{Single low-mass star}

Previous work generally assumed that N 66, irrespective of its peculiarities, belongs to the category of Planetary Nebulae, the central star being of low mass in an evolutionary phase after the Asymptotic Giant Branch (AGB). However, the luminosity of $\mathrm{N} 66$ (in its quiet state), $\log L / L_{\odot} \approx 4.6$, is exceptionally high for a post-AGB star or central star of a planetary nebula. Its luminosity corresponds to a core mass of about $1.2 M_{\odot}$ on a standard post-AGB track. The bulk of CSPN is compatible with theoretical post-AGB tracks for about $0.6 M_{\odot}$, in nice agreement with the preferred final mass of white dwarfs. Those standard tracks predict a hydrogen-rich surface composition, and might not apply to hydrogen-deficient stars. However, for a few hydrogen-deficient (i.e. [WC] type) central stars of known distance, empirical luminosities have been established (cf. Fig. 8). Obviously, they are not systematically brighter than hydrogenrich central stars, none of them exceeding $\log L / L_{\odot}=4.0$. The Eddington limit requires a minimum stellar mass of $0.7 M_{\odot}$ for the quiet-state luminosity. For the outburst, one must accept that the peak luminosity exceeds the Eddington limit unless the stellar mass is higher than $5 M_{\odot}$.

Post-AGB tracks with N 66's luminosity, such as the $1.2 M_{\odot}$ track from Paczyński (1970) shown in Fig. 8, cross the HRD extremely fast. Depending on the specific model, the time since leaving the AGB might be too short for ionizing the nebula of N 66 which, moreover, shines definitely since its discovery 50 years ago.

The surface composition is a further severe constraint to be discussed. The observed abundances, i.e. helium dominance, a rest of hydrogen and depleted carbon, are typical for partially $\mathrm{CNO}$ processed matter. Because of the bottleneck in the CNO cycle, most initial carbon is transformed into nitrogen soon after the burning started, while some hydrogen might be left when burning is terminated before the fuel is exhausted. However, in a post-AGB star there is only completely CNO processed material to be found below the (very thin) hydrogen burning shell. In order to explain the hydrogen observed in the atmosphere of $\mathrm{N} 66$, one might postulate that $\mathrm{CNO}-$ burnt (hydrogen-free) material might have been mixed with unburnt matter from outer layers. However, this would inevitably contaminate the CNO-burnt matter with a corresponding fraction of the initial carbon abundance, in contrast to the observed $\mathrm{C}$ depletion.

Another problem is why the hydrogen-rich envelope should mix with CNO processed layers. It is usually assumed that hydrogen-deficient central stars are produced when convection from a helium shell flash reaches the surface. This might happen either in a "final thermal pulse" at the AGB, or in form of a "(very) late thermal pulse" by which a post-AGB star is "born again" at the AGB for a second time (for a discussion, see e.g. Blöcker 2001; Herwig 2001; Koesterke 2001). Górny \& Tylenda (2000) have shown that the majority of Planetary Nebulae with [WC] type central stars are in conflict with the "born-again" scenario.

No matter which particular scenario actually applies to hydrogen-deficient central stars, it obviously brings the carbonrich products of helium burning from the intershell to the surface and thus produces [WC]-type central stars. The heliumrich surface composition of $\mathrm{N} 66$ seems to be a very rare or even unique exception. Vassiliadis (1996) discussed if the brightness outburst of $\mathrm{N} 66$ might reflect a late thermal pulse caught in the very act, but concluded that those delayed He-shell flashes are not very likely to occur for central stars with such relatively high mass, and moreover the brightness should have been raising more suddenly than observed.

\subsection{Single massive star}

Alternatively, we may discuss the possibility that $\mathrm{N} 66$ is in fact a massive Wolf-Rayet star of the nitrogen subclass (WN). Main problem of this hypothesis is that N66 is less luminous than any known massive WN (see Fig. 8). The least-luminous galactic WN analyzed in the comprehensive sample by Hamann \& Koesterke (1998a) is WR 2 with $\log L=5.0$ - also much too low compared to standard evolutionary tracks for massive stars, 
which are predicted to explode as a supernova while being red at those small masses. Following the mass-luminosity relation for core-burning helium stars from Langer (1989), N 66 would have a mass of $5.9 M_{\odot}$. In favor of this scenario, the peak luminosity at outburst remains just within the Eddington limit. Massive WN can show surface abundances as observed in N 66, but do not provide an explanation for the dramatic outburst. They sometimes possess circumstellar nebulae.

An argument against N 66 being a massive star comes from the terminal wind velocity. The escape velocity from a star with $5.9 M_{\odot}$ and the radius of $\mathrm{N} 66$ as determined $\left(0.52 R_{\odot}\right.$ outside outburst) is about $2000 \mathrm{~km} \mathrm{~s}^{-1}$, and we would expect a radiation-driven wind being 2-3 times faster than observed. On the other hand, assuming N 66 being a low-mass star of 1.2 $M_{\odot}$ results in $v_{\mathrm{esc}}=940 \mathrm{~km} \mathrm{~s}^{-1}$, much better in line with the observed wind velocities. A confusing fact is, that the terminal wind velocity during outburst is higher than in the quiet state, in contradiction to the correlation with $v_{\text {esc }}$ predicted by the radiation-driven wind theory (Castor et al. 1975).

So far we conclude, that we have no scenario at hand which could explain N 66 from single star evolution. Therefore we must consider a close-binary origin.

\subsection{Double-degenerate merger}

In two recent papers, Saio \& Jeffery $(2000,2002)$ investigated the merging of two white dwarfs and conclude that this is the best viable model for the creation of extreme helium stars. For a substantial fraction of close white dwarf binaries, the orbit decays within the Hubble time as a consequence of gravitational radiation or magnetic-wind braking. Finally, the less massive companion will fill its Roche lobe. After being disrupted by tidal forces, it will form a disc from which the more massive companion accretes the material.

The merging of two white dwarfs with helium cores (of, e.g., $0.4 M_{\odot}$ each) has been studied by Saio \& Jeffery (2000). When the accreting WD has reached a specific total mass, helium burning is ignited in a shell flash and the star becomes a yellow giant. After the accretion phase, the helium burning slowly proceeds inward with weaker shell flashes repeating on a time scale of a few thousand years. The final product is a helium star sitting close to the helium main sequence - just as observed for N66. However, the luminosity of these mergers is quite low $\left(10^{3} L_{\odot}\right.$, roughly). Thus this scenario could only apply to N66 if we just catch it in the phase of a bright flash. In order to explain the small hydrogen rest found in the extreme helium star V652 Her, Saio \& Jeffery (2000) speculate that some of the hydrogen rich envelopes of the progenitor white dwarfs could be expelled outwards during the initial dynamical phase of merging, and settle later on the surface of the merged product. This could also explain the surface hydrogen we observe in $\mathrm{N}$ 66. If the former WD hydrogen envelopes have been cleaned from metals by gravitational settling, carbon contamination could be avoided.

A different scenario applies if the pair of merging white dwarfs consists of one with $\mathrm{He}$ and one with $\mathrm{CO}$ core (Saio \& Jeffery 2002). Like in the $\mathrm{He}+\mathrm{He}$ case, helium shell burning is ignited during the accretion phase. By this, the star becomes a yellow giant of quite high luminosity (about $10^{4.4} \mathrm{~L}_{\odot}$ in their example with $0.6 M_{\odot}$ plus $0.4 M_{\odot}$ progenitors), comparable with what we see in $\mathrm{N} 66$ now. The merger then contracts at constant luminosity, similar to normal post-AGB evolution, and enters finally the WD cooling sequence.

Although those merger models are still highly simplified, similarities with N 66 are quite intriguing. But unfortunately these scenarios do not comply with the existence of the nebula. A severe contradiction arises from the $0.6 M_{\odot}$ of normally composed nebular matter. The merged white dwarfs cannot provide such an amount of hydrogen.

Having encountered now severe contradictions with all single-star models for N66, namely the low-mass single star evolution, the high-mass single star evolution, and the doubledegenerate merger scenario, we might discuss the hypothesis that $\mathrm{N} 66$ is at present still in a binary state.

We have checked our ample data base of more than a dozen of optical (ground-based) spectra from different epochs (see Table 1) for any radial velocity variations which could indicate that N66 is (still) a close binary system. No significant changes of the radial velocity could be found in our intermediate-resolution (2-3 $\AA$ ) spectra. However, the accuracy of that search is limited by the broadness of the stellar lines due to the atmospheric expansion, and also by some intrinsic variability of those line profiles. Between spectra from the same or from consecutive nights the intrinsic line variations are smallest, and we could exclude radial velocity differences of more than $50 \mathrm{~km} \mathrm{~s}^{-1}$ on that time scale.

In the kinematical study of its bipolar morphology, Peña et al. (2003) suggested that N 66 is seen almost pole-on. Hence a small sini might additionally hide an orbital velocity from our detection.

In binary scenarios, we have again to distinguish between a possible low-mass and high-mass origin of the primary, respectively.

\subsection{Massive star binary}

We discuss first the high-mass case. If single, a star of initially $10 M_{\odot}$ would stay as a red supergiant till suffering a gravitational collapse (supernova). However, a low-mass close companion of, say, $1 M_{\odot}$, would be incorporated in a common envelope when the primary becomes a red supergiant. By the energy input of the secondary, the hydrogen-rich envelope of the red supergiant can be expelled and form a nebula. The primary then remains as basically a helium star at the helium main sequence - as N 66 is found at a position which corresponds to a present mass of $5.9 M_{\odot}$ (cf. Sect. 5.2). The unevolved lowmass secondary survives unaffected, but due to the mass ratio, the radial velocity variation of the primary would be undetectably small. We conclude that the massive-star, post-redsupergiant, post-common-envelope scenario provides a possible explanation for the nature of $\mathrm{N} 66$, the only remaining objection being the $v_{\infty}$ over $v_{\text {esc }}$ ratio (cf. Sect. 5.2). Surface abundances as observed follow naturally from this scenario, but no explanation is provided for the observed brightness outburst. 
In Paper I we tended to assume that the latter was a kind of atmospheric instability, similar to Luminous Blue Variables, but the strong variation in luminosity (at constant effective temperature) which we derived now from the spectral analyses does not corroborate that assumption.

\subsection{Low-mass binary}

Alternatively, N66 might be a low-mass binary system presently undergoing strong mass transfer. A white dwarf, originally the primary of the system, accretes matter from a nondegenerate star, probably in a second mass exchange. If the accreted matter contains enough hydrogen, this might be burned more or less smoothly in the WD's outer layers. In any case, the accreted helium (and the helium possibly produced by hydrogen burning) will feed a helium-burning shell. If the nuclear energy production leads to a higher luminosity than the Eddington limit, accretion would probably stop. The (quiet state) luminosity of N 66 is below the Eddington limit for any WD mass higher than $0.7 M_{\odot}$. If the accreted matter is composed as we have determined for the wind, hydrogen burning could produce the major part of the nuclear luminosity. In order to fuel the observed $\log L / L_{\odot}=4.6$, mass has to be accreted with a rate of about $\log \left[\dot{M} /\left(M_{\odot} \mathrm{yr}^{-1}\right)\right]=-5.7$, which is interestingly of the same order of magnitude as the observed (quiet state) massloss rate.

In this scenario, the stellar wind of N 66 probably consists of the freshly accreted material, possibly mixed with (heliumrich?) matter from the WD's envelope. In any case, from its carbon deficiency follows that the material transferred has undergone partial CNO burning before. This puts some constraint to the history of the mass-exchanging system, but is not impossible to imagine.

Note that this scenario differs from usual Cataclysmic Variables (CVs) because of the much higher mass accretion rate. Such high rates are predicted for the early phase of mass transfer, when the donor star is still more massive than the gainer and, therefore, the mass transfer leads to a shrinking orbital separation (Langer et al. 2002). This phase is much shorter than later phases of low accretion usually observed in CVs.

The spectrum of N 66 is clearly that of a mass-loosing star, not that of an accretion disk. This is not surprising, as a disk with any reasonable accretion rate is outshined by such a bright star. Therefore, an unstable disk luminosity like in dwarf novae cannot account for the outburst of N 66. On the other hand, a thermonuclear explosion like in classical novae also does not match to N 66. In classical novae the accretion rate is too small to sustain steady-state burning. Therefore, while being quiet they are much dimmer than $\mathrm{N} 66$. until the accumulated hydrogen detonates suddenly.

Detailed evolutionary calculations for helium-accreting white dwarfs have been performed in Potsdam, but only little has been published yet (Langer et al. 2002). Those calculations show amazing similarities with N66. White dwarfs accreting with a high rate do not suffer from a shortage of fuel, unlike CVs, but sustain steady shell burning like post-AGB stars. However, they are more luminous than normal post-AGB stars of the same mass, because their degenerate core is even more compact. They develop helium shell flashes (when the helium burning shell becomes too thin), during which the luminosity reaches the Eddington limit.

Such a helium shell flash provides a possible explanation for the outburst of N 66. From comparing the observed outburst duration (of the order of years) with the Helmholtz-Kelvin timescale we can infer that the outburst only involves the outermost layers of $\mathrm{N} 66\left(10^{-3} M_{\odot}\right)$. In the calculations by Langer et al. (2002) the thermal pulses repeat on a timescale of $10^{3} \mathrm{yr}$, depending on the particular model parameters. The nebular ejection(s), some 500 to 5000 years ago, might have occurred at previous thermal pulses. The matter accreted (and ejected) that time was obviously still hydrogen rich. This would indicate that N 66 has entered the stage of helium accretion only recently.

The stellar radius of $\mathrm{N} 66$ is much bigger than that of a white dwarf. This is probably a further consequence of the high accretion rate, which exceeds the steady-state burning rate and therefore builds up an extended envelope. In order to be consistent with our analysis of the outburst, we must assume that this envelope has damped out most of the thermal pulse in a way that the radius increased only moderately, while the effective temperature remained roughly constant.

As expected from its relatively low surface temperature, N 66 is not detected as an X-ray source. In contrast, the central star of N67 in the SMC has been identified with the "supersoft X-ray source" 1E00056.8-7154 (Wang 1991). Very similar to N66, N67 is a high-excitation planetary nebula with the same, exceptionally high luminosity. Such objects are tentatively identified with accreting white dwarfs just as in our scenario (Greiner 2000). N 66 differs from those objects by its larger radius.

\section{Conclusions}

Spectra of N 66 from different epochs have been analyzed in detail by means of the most advanced non-LTE models for expanding stellar atmospheres. The main results are: a high luminosity of $\log L / L_{\odot}=4.6$ before and after the outburst, which climbed up by almost a factor of ten in 1994 for about one year, while the effective temperature of about $112 \mathrm{kK}$ remained constant. The mass loss rate increased from $10^{-5.7} M_{\odot} \mathrm{yr}^{-1}$ in the quiet state to $10^{-5.0} M_{\odot} \mathrm{yr}^{-1}$ during the outburst. The chemical composition of the stellar atmosphere is that of incompletely CNO-processed matter: it is dominated by helium with a rest of hydrogen, nitrogen being slightly enhanced and carbon strongly depleted. The bipolar nebula, ejected only a few thousand years ago, contains about 0.6 solar masses of hydrogenrich matter.

We extensively discussed possible scenarios for the nature and evolutionary origin of N66. None of the scenarios remained without any contradiction to at least one of the observational facts. A low-mass single star after the AGB is in conflict with surface abundances. Massive, i.e. non-degenerate single stars are not predicted to reach this part of the HR diagram. A merger from two white dwarfs can hardly have produced the hydrogen-rich nebula. A massive star, which lost its hydrogen 
envelope in a recent common-envelope phase with a less massive companion, is in conflict with the too low terminal wind speed and offers no explanation for the observed outburst. For a white dwarf accreting mass from a companion, i.e. a kind of cataclysmic variable, the parameters are quite unusual.

We think the two last-mentioned scenarios, a massive star after a common-envelope phase, and a helium-accreting white dwarf, pose the least severe contradictions. Most exciting is the speculation that $\mathrm{N} 66$ might be a massive white dwarf accreting matter in a close-binary system. Its present, high accretion rate would bring it to the Chandrasekhar limit within a few hundred thousand years. Thus N66 might be a candidate for a future type Ia supernova explosion in our cosmic neighborhood.

Acknowledgements. We thank Norbert Langer, Simon Jeffery and Falk Herwig for enlightening discussions about the evolutionary origin of N66. The comments of the referee, R. Tylenda, have been extremely useful. M.P. acknowledges financial support from DGAPA/UNAM (IN 114601) and CONACYT/Mexico (grant 32594-E). M.T.R. received partial support from FONDAP (15010003), a Guggenheim Fellowship, and Fondecyt (1010404).

\section{References}

Aller, L. H. 1984, Physics of thermal gaseous nebulae: Physical processes in gaseous nebulae (Dordrecht: Reidel)

Asplund, M., Lambert, D. L., Kipper, T., Pollacco, D., \& Shetrone, M. D. 1999, A\&A, 343, 507

Benedict, G. F., McArthur, B. E., Fredrick, L. W., et al. 2002, AJ, 124, 1695

Blades, J. C., Barlow, M. J., Albrecht, R., et al. 1992, ApJ, 398, L41

Blöcker, T. 1993, Acta Astron., 43, 305

Blöcker, T. 1995, A\&A, 299, 755

Blöcker, Th. 2001, Ap\&SS, 275, 1

Castor, J. I., Abbott, D. C., \& Klein, R. I. 1975, ApJ, 195, 157

Crowther, P. A., Hillier, J. D., \& De Marco, O. 2003, in Planetary nebulae and their Role in the Universe, ed. M. Dopita, \& S. Kwok, ASP Conf. Ser., in press

Dopita, M. A., Ford, H. C., Lawrence, C. J., \& Webster, B. L. 1985, ApJ, 296, 390

Dopita, M. A., Ford, H. C., Bohlin, R., Evans, I. N., \& Meatheringham, S. J. 1993, ApJ, 418, 804

Feast, M. M. 1988, in The Extragalactic Distance Scale, ed. S. van den Bergh, \& C. J. Pritchet (San Francisco: ASP), ASP Symp., 100, 4
Górny, S. K., \& Tylenda, R. 2000, A\&A, 362, 1008

Gräfener, G., Koesterke, L., \& Hamann, W.-R. 2002, A\&A, 387, 244

Gräfener, G., Hamann, W.-R., \& Peña, M. 2003, in Planetary nebulae and their Role in the Universe, ed. M. Dopita, \& S. Kwok, ASP Conf. Ser., in press

Greiner, J. 2000, New Astron. 5, 137

Groenewegen, M. A. T., \& Lamers, H. J. G. L. M. 1989, A\&A, 221, 78

Hamann, W.-R. 2003, in Stellar Atmosphere Modelling, ed. I. Hubeny, D. Mihalas, \& K. Werner, ASP Conf. Ser., in press

Hamann, W.-R., \& Koesterke, L. 1998a, A\&A, 333, 251

Hamann, W.-R., \& Koesterke, L. 1998b, A\&A, 335, 1003

Henize, K. G. 1956, ApJS, 2, 315

Herwig, F. 2001, AP\&SS, 275, 15

Howarth, I. D. 1983, MNRAS, 203,301

Koesterke, L. 2001, AP\&SS, 275, 41

Langer, N. 1989, A\&A, 210. 93

Langer, N., Yoon, S.-C., Wellstein, S., \& Scheithauer, S. 2002, in The Physics of Cataclysmic Variables and Related Objects, ed. B. T. Gänsicke, K. Beuermann, \& K. Reinsch, ASP Conf. Proc., 261, 252

Leuenhagen, U., Hamann, W.-R., \& Jeffery, C. S. 1996, A\&A, 312, 167

Monk, D. J., Barlow, M. J., \& Clegg, R. E. S. 1988, MNRAS, 234, 583

Nail, V. M., \& Shapley, H. 1955, Proc. Natl. Acad. Sci., 41, 685

Paczyński, B. 1970, Acta Astron., 20,47

Peña, M., \& Ruiz, M. T. 1988, Rev. Mex. Astron. Astrofis., 16, 55

Peña, M., Torres-Peimbert, S., Peimbert, M., Ruiz, M. T., \& Maza, J. 1994, ApJ, 428, L9

Peña, M., Peimbert, M., Torres-Peimbert, S., Ruiz, M. T., \& Maza, J. 1995, ApJ, 441, 343

Peña, M., Hamann, W.-R., Koesterke, L., et al. 1997, ApJ, 491, 233 (Paper I)

Peña, M., Hamann, W.-R., Ruiz, M.T., Peimbert, A., \& Peimbert, M. 2003, ApJ, submitted

Saio, H., \& Jeffery, C. S. 2000, MNRAS, 313, 671

Saio, H., \& Jeffery, C. S. 2002, MNRAS, 333, 121

Schaller, G., Schaerer, D., Meynet, G., \& Maeder, A. 1992, A\&AS, 96, 269

Schmutz, W., Hamann, W.-R., \& Wessolowski, U. 1989, A\&A, 210, 236

Seaton, M. J. 1979, MNRAS, 187, 73P

Torres-Peimbert, S., Peimbert, M., Ruiz, M. T., \& Peña, M. 1993, in Planetary Nebulae, ed. R. Weinberger, \& A. Acker (Dordrecht: Kluwer), IAU Symp., 155, 584

Vassiliadis, E. 1996, ApJ, 465, 748

Wang, Q. 1991, MNRAS, 252, 47 